\title{
ALICE Dimuon Spectrometer
}

Hard, penetrating probes, such as heavy quarkonium states, provide an essential tool to study the early and hot stage of heavy-ion collisions. In particular they are expected to be sensitive to Quark-Gluon Plasma formation. In the presence of a deconfined medium (i.e. QGP) with high enough energy density, quarkonium states are dissociated because of colour screening. This leads to a suppression of their production rates. At the high LHC collision energy, both the charmonium states $\left(\mathrm{J} / \Psi\right.$ and $\left.\Psi^{\prime}\right)$ as well as the bottomonium states $\left(\Upsilon, \Upsilon^{\prime}\right.$ and $\left.\Upsilon^{\prime \prime}\right)$ can be studied. The Dimuon spectrometer is optimized for the detection of these heavy quark resonances.

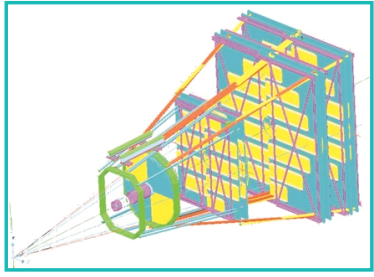

Geometry Monitoring System

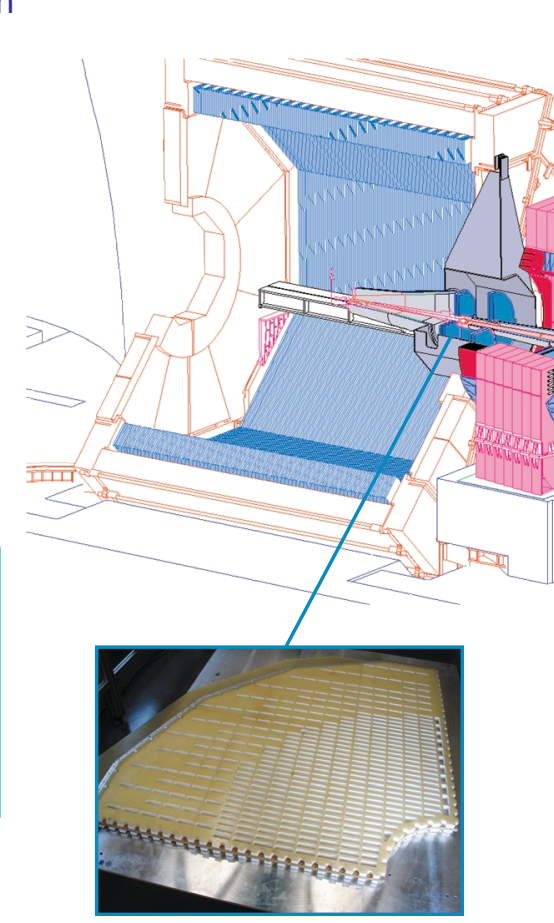

Quadrant of the small chambers
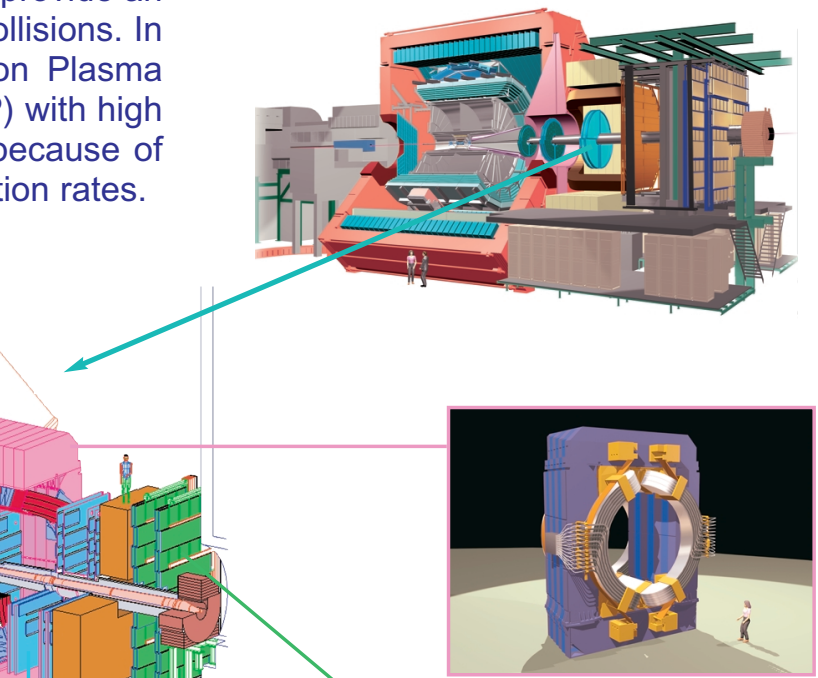

Dipole Magnet

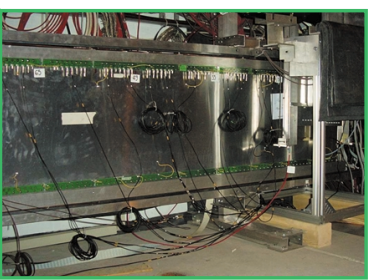

Resistive Plate Chamber for the trigger system

The ALICE Forward muon spectrometer

The ALICE forward muon spectrometer will study the complete spectrum of heavy quarkonia $\left(J / \Psi, \Psi^{\prime}, Y, Y^{\prime}, Y^{\prime \prime}\right)$ via their decay in the $\mu^{+} \mu^{-}$channel. The spectrometer acceptance covers the pseudorapidity interval $2.5 \leq \eta \leq 4$ and the in the $\mathrm{J} / \Psi$ region and about $100 \mathrm{MeV}$ close to the $Y$. These values allow to resolve and measure individually all five resonance states

The main components of the spectrometer are shown on the following sketch.

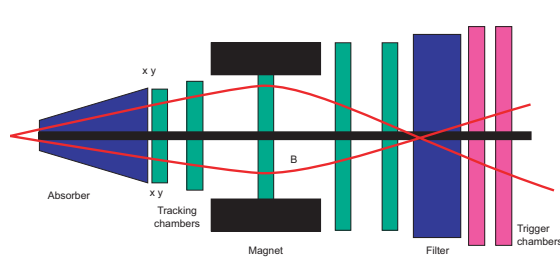

The front absorber suppresses all particles except muons coming from carbon and concrete in order to limit the mullte scattering and the beam shield protects the chambers from background originating from particles at small angles. It is made of tungsten, lead and stainless stee to minimize the background arising collision and from their showers produced in the beam pipe and in Basic principle of the Dimuon spectrometer: an absorber to filter the background, a set
of tracking chambers before, inside and atter the magnet and a set of trigger chambers

The tracking system is made of 10 cathode pad/strip chambers arranged in 5 stations of 2 chambers each. The spatia esolution should be better than $100 \mathrm{~mm}$ and all the chambers are made of composite material $\left(<3 \% \mathrm{X}_{0}\right.$ per chamber) minimize the scattering of the muons in order to obtain the required
of $5 \%$ the full set of chambers has more than 1 million channels.

The trigger system is designed to select heavy quark resonance the $p_{t}$ of the two individual muons. The 4 planes of RPCs (Resistive Plate Chambers) arranged in 2 stations and positioned behind a transverse momentum of each The spatial resolution should be better than $1 \mathrm{~cm}$. Special front-end electronics have been designed to

for the identification of the bunch crossing.

The dipole magnet is positioned at about $7 \mathrm{~m}$ from the interaction vertex and it is one of the biggest warm dipoles in the world (free gap between poles $\approx 3 \mathrm{~m}$, height of the yoke $~ 9 \mathrm{~m}$ ). The magnetic field $\left(B_{\text {nom }}=0.7 \mathrm{~T}, 3 \mathrm{Tm}\right.$ field integral) is defined by the requirements on the mass resolution.
Physics programme

Heavy quarkonia

The production of all the quarkonia from the $\Psi$ and $\Upsilon$ families will be studied:

- as a funciton

patterns;

- with respect to the reaction plane (determined with other ALCE detectors, e.g. the PMD) to unravel Glauber and comover absorption for different colliding nuclei (e.g. Pb-Pb and Ar-Ar) to investigate the dependence of quarkonia yields as a function of the system size; reference for nucleus-nucleus.

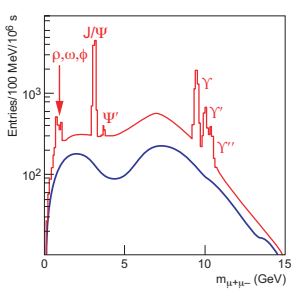

Open Charm and Beauty

In the dimuon invariant mass spectrum, the quarkonia signals will be sitting on top of a continuum mainly coming from open charm and bottom decay. This opens the possibility of studying the production of open charm and beauty in paralle

- open charm and beauty represent the most natural normalization of the quarkonia signals; of open flavours cross-sections are expected to shed light on production mechanisms for heavy quarkonia other than direct hard-scattering

Run conditions and statistics

The following data taking scenario is at present foreseen in the first LHC years:

- p-p runs at $\sqrt{ } \mathrm{s}=14 \mathrm{TeV}$ at $\mathrm{L}=310^{30} \mathrm{~cm}^{-2} \mathrm{~s}^{-1}$

- $\mathrm{Pb}-\mathrm{Pb}$ runs at $\mathrm{L}=1027 \mathrm{~cm}^{-2} \mathrm{~s}^{-1}$

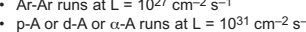

In addition to this program, later options will be considered depending on the outcome of the first years.

(ith i.e. about one collisions as well as for $p-p$ collisions. For the $\gamma$, an excellent signal to background ratio (typically of the order of 10) is expected. 